\title{
Monitoring radiation damage in the ATLAS Silicon Tracker
}

\author{
Stephen Gibson* on behalf of the ATLAS Collaboration. \\ CERN - Geneva - Switzerland. \\ E-mail: Stephen.Gibson@cern.ch
}

\begin{abstract}
The record breaking instantaneous luminosities of $10^{33} \mathrm{~cm}^{-2} \mathrm{~s}^{-1}$ recently surpassed at the Large Hadron Collider generate a rapidly increasing particle fluence in the ATLAS silicon tracker. As the radiation dose accumulates, the first effects of radiation damage are now observable in the silicon sensors. A regular monitoring program has been conducted and reveals an increase in the silicon leakage current, which is found to be correlated with the rising radiation dose recorded by independent sensors within the Inner Detector volume. Such measurements are useful to validate the digitization model that has been developed to simulate radiation damage effects, including charge trapping, electric field modification and realistic signal induction on the electrodes. In the longer-term crystal defect formation in the silicon bulk is expected to alter the effective doping concentration, producing type-inversion and ultimately an increase of the voltage required to fully deplete the sensor. Together with measurements of the depletion voltage, we present a track-based method that has been developed to estimate the depth of the depleted zone, allowing a continuous monitoring of the sensor performance. In the Pixel Detector, Lorentz angle effects are reduced by a novel implementation of the method in the longitudinal pixel dimension.
\end{abstract}

10th International Conference on Large Scale Applications and Radiation Hardness of Semiconductor Detectors,

6-8 July 2011

Firenze, Italy

\footnotetext{
* Speaker.
} 


\section{Introduction}

The innermost layer of the silicon tracker of the ATLAS experiment [1] is designed to tolerate a radiation dose of $500 \mathrm{kGy}$ for a fluence of $10^{15} 1 \mathrm{MeV} \mathrm{n}_{\mathrm{eq}} \mathrm{cm}^{-2}$, during operation in Phase I at the Large Hadron Collider, CERN. The integrated luminosity of $\sim 1.3 \mathrm{fb}^{-1}$ acquired before the RD11 conference on 6 July 2011, corresponds to a fluence of $3.1 \times 10^{12} 1 \mathrm{MeV} \mathrm{n}_{\mathrm{eq}} \mathrm{cm}^{-2}$ at the innermost tracking layer. Even though this is a small fraction of the full dose and is well before the regime for type-inversion ${ }^{1}$ the first signs of radiation damage are now observable in the silicon tracker and initial measurements of these effects are reported here.

After introducing the detector layout, this section describes the main effects of radiation damage in silicon and how such effects are monitored in ATLAS. The voltage required to fully deplete the silicon is critical to the sensor performance and first measurements of changes in this parameter due to radiation damage in the Pixel Detector are described in Section 3, along with a novel track-based method to monitor the depletion depth in future after type-inversion.

\subsection{The ATLAS Inner Detector}

The trajectories of charged particles emerging from the ATLAS interaction point are measured by three sub-detectors, arranged as in Figure 1. The two silicon sub-detectors provide hermetic

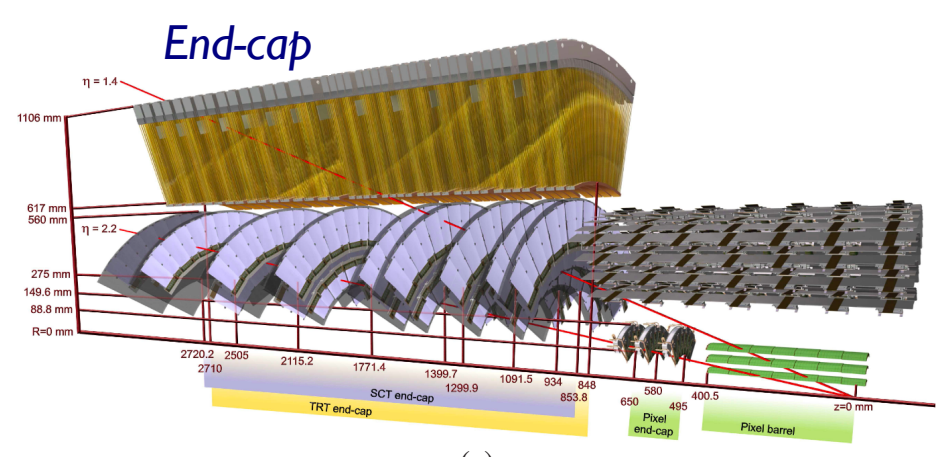

(a)

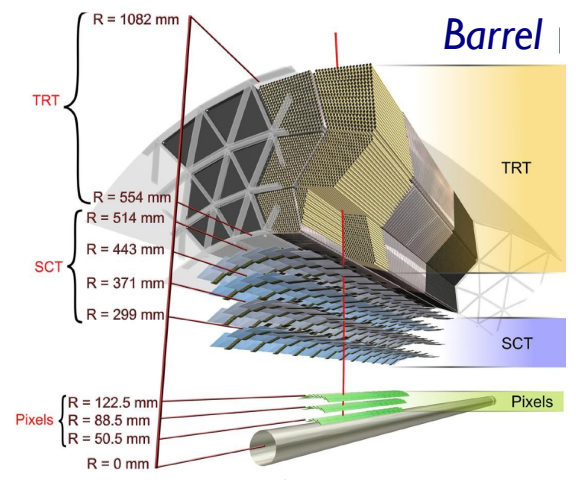

(b)

Figure 1: The End-cap (a) and Barrel (b) layout of the ATLAS Inner Detector.

particle tracking within the pseudorapidity range of $|\eta|<2.5$. At low radii, $50.5-149.6 \mathrm{~mm}$, highly granular coverage is provided by three layers of $\mathrm{n}^{+}$-in-n type silicon pixel sensors, with an intrinsic resolution of $10(115) \mu \mathrm{m}$ in $\mathrm{r} \phi(\mathrm{rz})$. Each module has $47232(328 \times 144)$ pixels with 46080 pixels bump-bonded to 16 front end chips, read out at a rate of $40-160 \mathrm{MHz}$ depending on the layer. At intermediate radii, $299-514 \mathrm{~mm}$, a particle track traverses at least four double-layered modules built from two single-sided silicon micro-strip sensors. A module comprises two wafer layers of 768 p-type strips on n-type silicon with an $80 \mu \mathrm{m}$ pitch, which are glued back-to-back at a stereo angle of $40 \mathrm{mrad}$ to give an intrinsic resolution of $17(580) \mu \mathrm{m}$ in $\mathrm{r} \phi(\mathrm{rz})$. At larger radii, 554 $1108 \mathrm{~mm}$, a system of straw-tubes provides particle identification based on transition radiation effects and improves the momentum resolution for the Inner Detector to the design of $\sigma\left(p_{T}\right) / p_{T}=$ $0.05 \% p_{T}[\mathrm{GeV} / \mathrm{c}] \oplus 1 \%$.

\footnotetext{
${ }^{1}$ the $\mathrm{n}$-type bulk becomes effectively p-type after a fluence of $2 \times 10^{13} 1 \mathrm{MeV} \mathrm{n}_{\mathrm{eq}} \mathrm{cm}^{-2}$ with optimal annealing.
} 


\subsection{Effects of radiation damage in silicon}

Bulk damage in silicon is expected to result in crystal defects that will alter the physical properties of the sensor and change the operating conditions:

- The introduction of acceptor centres will modify the doping concentration and lead to typeinversion, after which the voltage required to fully deplete the sensor will increase.

- Recombination / generation centres will increase the leakage current, affecting the power consumption and signal to noise.

- Charge trapping centres will reduce the charge collection efficiency and hence degrade the hit efficiency, track resolution and $b$-tagging performance.

\subsection{Radiation damage monitoring and simulation}

Regular monitoring of the effects of radiation damage in the ATLAS silicon tracker is critical for understanding and optimising the detector performance. The measurements also serve to validate the digitization model, which has been developed to include charge trapping, electric field modification and realistic signal induction on the electrodes. The aim of this model is to reproduce the observable effects of radiation damage and thus enable the Monte Carlo simulation of physics data to accurately describe the detector response during all phases of operation.

The first key observable effect is the increase in leakage current which is proportional to fluence. The leakage current is typically continuously monitored per module in the Pixel Detector and the Silicon Micro-strip Detector, while finer granularity is possible with dedicated calibration scans. The front end chip of the Pixel Detector has the capability to measure the leakage current per pixel, with a $0.125 \mathrm{nA}$ least significant bit and with a dynamic range optimised for the high irradiation fluence of $10^{15} 1 \mathrm{MeV} \mathrm{n} \mathrm{nq}_{\mathrm{e}} \mathrm{cm}^{-2}$. The per pixel method was just below sensitivity for the fluence at the time of the conference, therefore no results are shown from this on-chip measurement.

A second observable effect is the change in the voltage required to fully deplete the sensor. In the Pixel Detector this is regularly monitored during routine maintenance stops of the LHC using the cross-talk method described in Section 3, which is valid before type-inversion. Track-based methods have been developed to assess the depletion depth of the pixel sensors after type-inversion.

\section{Evolution of the Silicon Leakage Current}

\subsection{Leakage current in the Pixel Detector}

\subsubsection{Current Monitoring Boards}

The innermost Pixel Detector barrel layer is instrumented with Current Monitoring Boards (CMBs) [2] that enable the leakage current of individual modules (56 modules as of June 2011) to be measured with a precision approaching $\sim 10 \mathrm{nA}$. Figure 2(a) shows the raw currents recorded by the CMBs between March and June 2011. The increases in the measured currents are correlated with the rise in the integrated luminosity delivered to ATLAS.

The heat dissipated by the front end electronics is removed by an evaporative $\mathrm{C}_{3} \mathrm{~F}_{8}$ cooling system, which maintains a silicon temperature of well below $0{ }^{\circ} \mathrm{C}$ to mitigate radiation damage. 
The temperature measured by $10 \mathrm{k} \Omega \mathrm{NTC}^{2}$ thermistors placed on each module was relatively stable at an average of $-13{ }^{\circ} \mathrm{C}$ over the time period of the current measurements, as shown in Figure 2(b), with a few temporary excursions due to a cooling stoppage or Pixel Detector calibration scans.

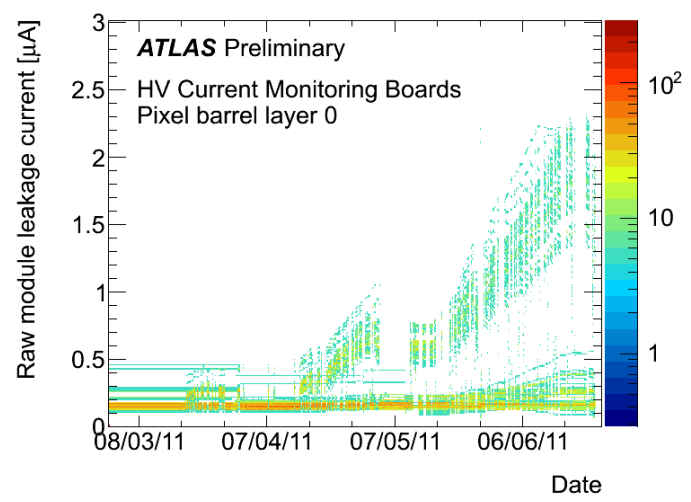

(a)

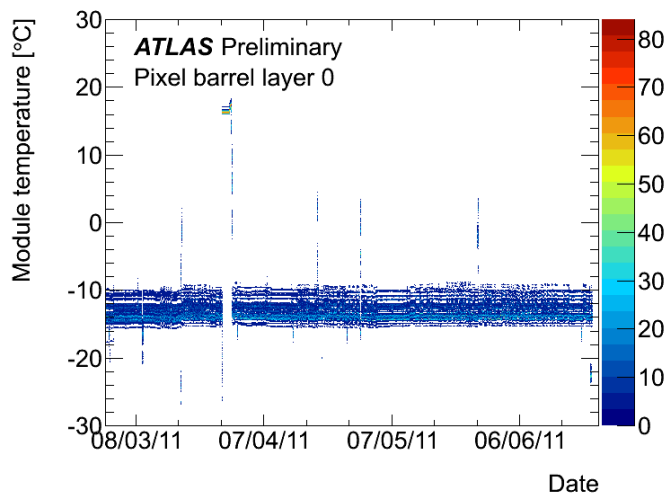

(b)

Figure 2: The uncorrected leakage current (a) of the innermost Pixel Detector barrel layer modules measured by the Current Monitoring Boards and the module temperature profile (b) over the same period.

The raw modules currents were corrected for pedestal current and scaled to a reference temperature of $-10{ }^{\circ} \mathrm{C}$ using the formula:

$$
I\left(T_{\mathrm{REF}}\right)=I(T)\left(\frac{T_{\mathrm{REF}}}{T}\right)^{2} \cdot \exp \left[-\frac{E_{g}}{2 k_{\mathrm{B}}}\left(\frac{1}{T_{\mathrm{REF}}}-\frac{1}{T}\right)\right]
$$

where $E_{g}$ is the silicon band gap, $1.21 \mathrm{eV}$ [3] and $k_{\mathrm{B}}$ is the Boltzmann constant.

A further, preliminary correction is applied for the beam ionisation current, $I_{\text {hit }}$, induced from charged particle hits in the modules:

$$
I_{\text {hit }}=N_{\text {bunches }} \cdot v_{\mathrm{LHC}} \cdot \text { Occ } \cdot C_{\mathrm{hit}}
$$

where, $N_{\text {bunches }}$ is the number of colliding bunches, $v_{\mathrm{LHC}}$ is the LHC revolution frequency, Occ is the pixel hit occupancy per module per colliding bunch crossing (average taken per barrel layer) and $C_{\text {hit }}$ is the charge per hit.

The average leakage current profile after the above corrections is shown in Figure 3. An approximately linear correlation is found versus integrated luminosity, suggesting that the fluence in the Pixel Detector is dominated by proton-proton collisions, rather than by other sources such as beam backgrounds. The calibration of the $56 \mathrm{CMBs}$ is ongoing and requires measurements with and without LHC beam. Measurements of the current data from all modules are described below.

\subsubsection{Monitoring half-stave $\mathrm{HV}$ currents}

In the Pixel Detector barrel one ISEG unit supplies power to a half-stave, consisting of either 6 or 7 pixel modules. The current can be monitored with a precision of $\sim 80 \mathrm{nA}$ per half-stave. The above procedure was applied to correct the measured leakage currents for the module temperature,

\footnotetext{
${ }^{2}$ Negative Temperature Coefficient thermistors loaded on the kapton circuit and in thermal contact with the silicon.
} 


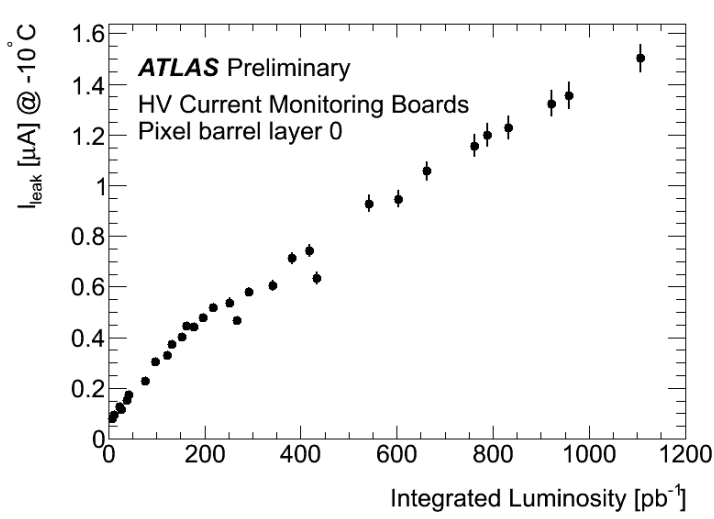

(a)

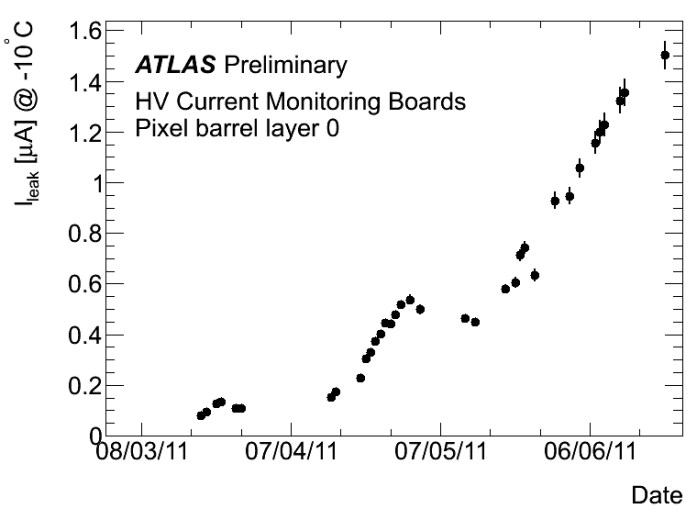

(b)

Figure 3: Increase in the leakage current per module vs the integrated luminosity (a) and the date (b) averaged over the 56 modules with Current Monitoring Boards in the innermost Pixel Detector barrel layer.

the beam induced current and the number of modules per half-stave. An average was then taken over each barrel layer and the increase in leakage current is found to be correlated with the integrated luminosity, as plotted in Figure 4(a). The same data are plotted vs date in Figure 4(b). A prediction is also shown based on the measured luminosity profile, the radial fluence expectation from FLUKA simulations of minimum bias proton-proton events and the damage constant, $\alpha$ [4]. Reasonable agreement of the measured current is found with the prediction from the simulated fluence in the three barrel layers of the Pixel Detector.

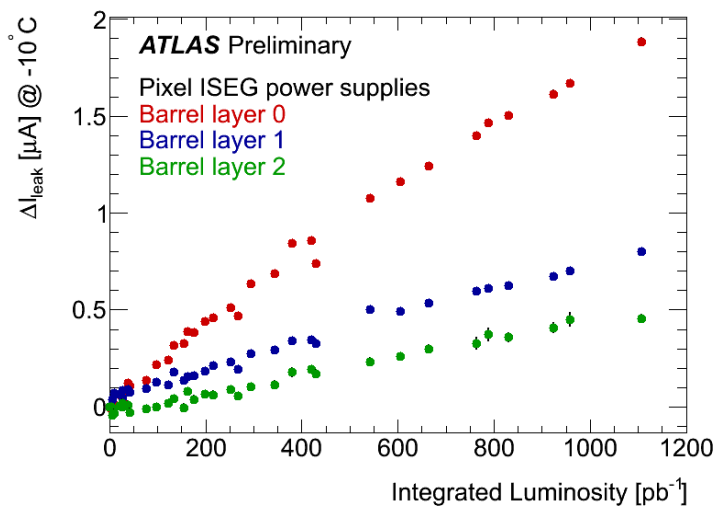

(a)

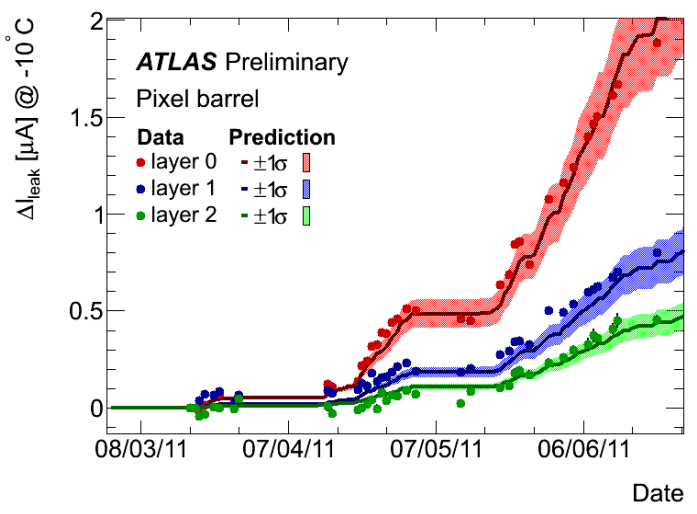

(b)

Figure 4: Increase in the module average leakage current vs integrated luminosity (a) and vs date (b) in the Pixel Detector barrel layers.

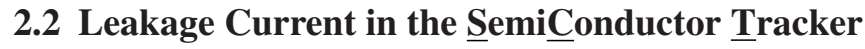

The leakage currents in the modules of the silicon micro-strip tracker have been monitored constantly since the start of ATLAS running. Dedicated measurements are also taken in regular maintenance days without the LHC beam and with the optical alignment system turned off to eliminate induced photocurrent on some modules. The resolution of $10 \mathrm{nA}$ enabled an early observation of the increase in leakage current in the Barrel SCT at the end of 2010 proton run, as in Figure 5. 
Even after only $48.6 \mathrm{pb}^{-1}$ of integrated luminosity, a clear radial dependence of the leakage current increase was observed between the four barrel layers, indicative of radiation damage.
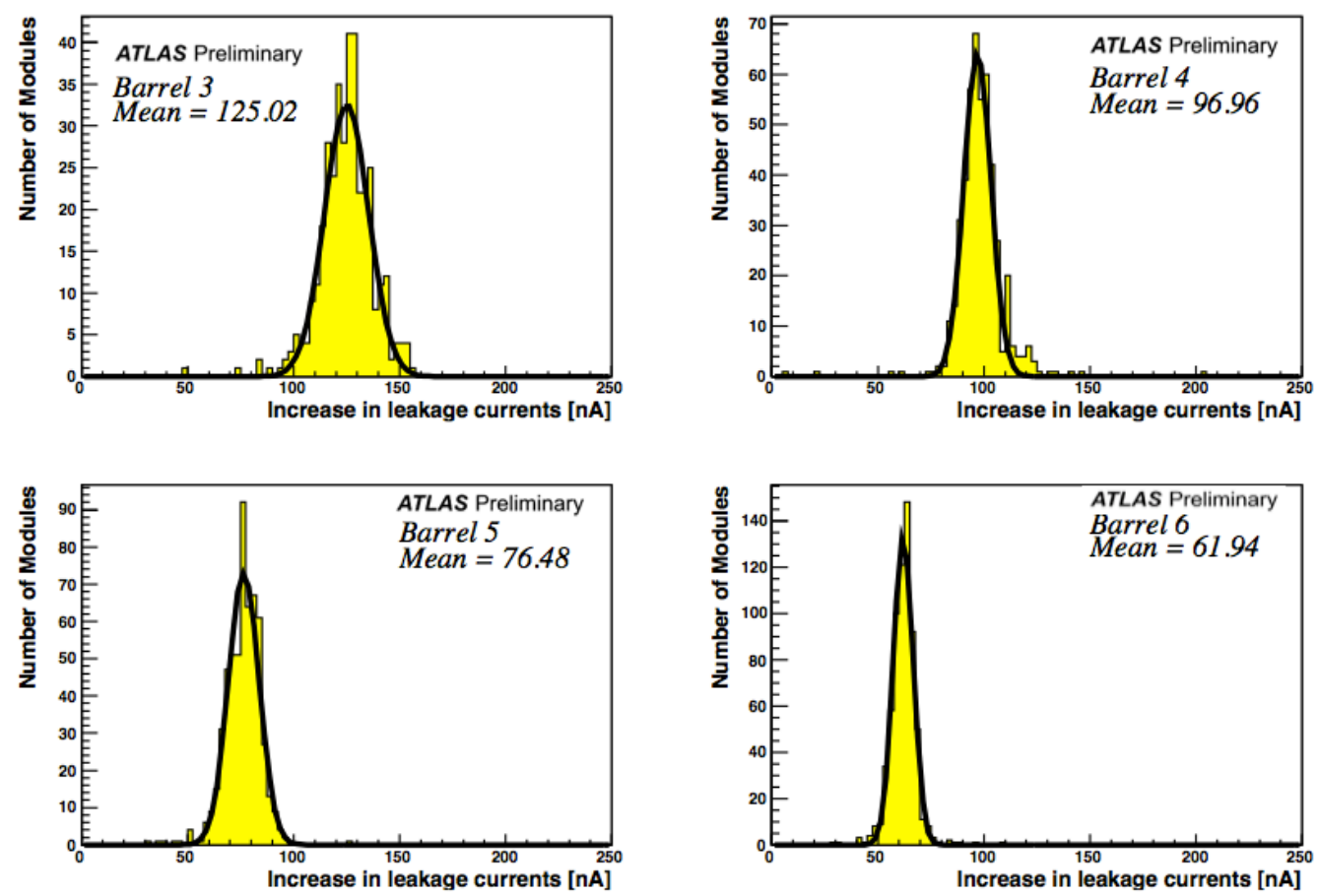

Figure 5: Increase in the average module leakage current in the four SCT barrel layers up to the end of proton running in 2010. Barrels 3, 4, 5 and 6 have radii of 299, 371, 443 and $514 \mathrm{~mm}$ respectively.

The mean values from similar measurements performed at intervals up to the conference date are shown by the points in Figure 6(a). The leakage current is scaled to the reference temperature of $-10^{\circ} \mathrm{C}$. A prediction is shown based on the total $7 \mathrm{TeV}$ luminosity profile and proton-proton minimum bias FLUKA simulations, taking self-annealing effects into account, including over the winter shutdown. The prediction uncertainties are mostly due to errors in the fraction of the slowest annealing component (11\%) and luminosity measurement (4.5\% in 2011). Any systematic uncertainty from the FLUKA simulation is not included. In Figure 6(b) the fluence estimate from the measured SCT leakage current is compared as a ratio with the fluence predicted from FLUKA simulation over one quadrant of the SCT in the R-Z plane. The good agreement indicates that the simulated fluences at the barrel are accurate to within $10 \%$ or so. A larger difference is found in the inner end-cap regions.

\section{Depletion Depth Monitoring}

The voltage required to fully deplete the silicon sensor $V_{\text {dep }}$ is critical to the performance of the detector, as the depleted depth determines the collected charge. Initially $\mathrm{V}_{\text {dep }}$ is expected to decrease up to type-inversion of the silicon bulk, after which $V_{\text {dep }}$ will constantly increase with the irradiation fluence. For the Pixel Detector, the present bias voltage of $150 \mathrm{~V}$ can be increased up to the existing power supply rating of $600 \mathrm{~V}$, which is foreseen in the later years of operation. 


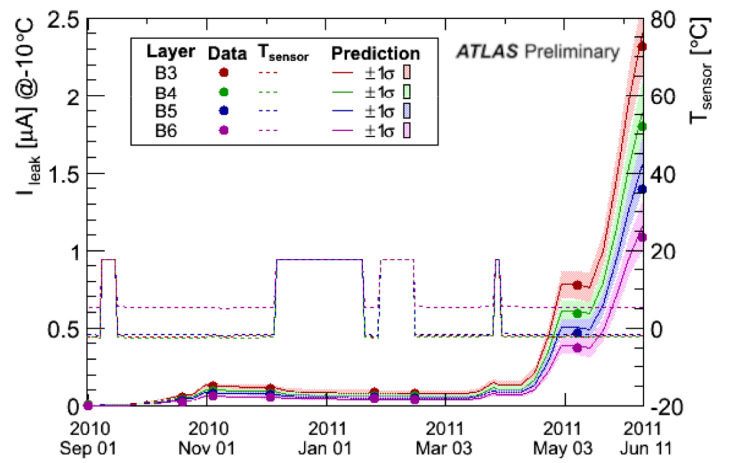

(a)

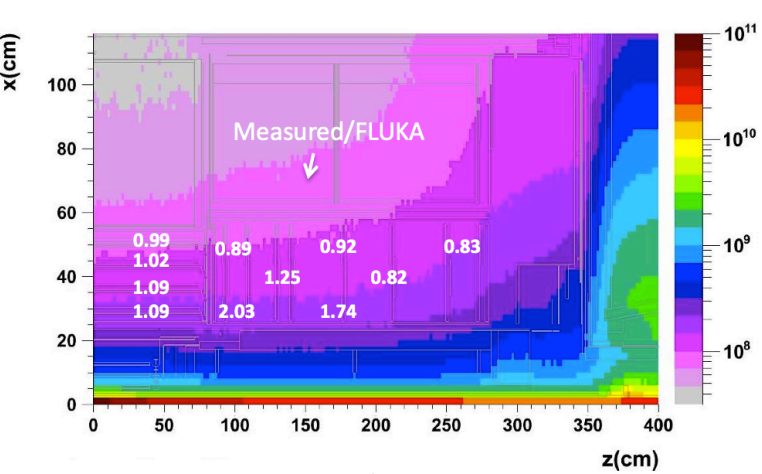

(b)

Figure 6: The SCT module leakage current measurement compared with the prediction from FLUKA simulation (a) and the FLUKA simulated fluence $\left[1 \mathrm{MeV} \mathrm{n}_{\mathrm{eq}} \mathrm{cm}^{-2}\right]$ (b); the overlaying numbers are the ratio of the fluence calculated from the measured SCT leakage current to the FLUKA prediction.

After type-inversion the depletion zone will grow from the read-out plane, enabling operation of the Pixel Detector even when partially depleted.

\subsection{Depletion voltage measurements}

The depletion voltage in the Pixel Detector is regularly monitored during scheduled downtime of the LHC. A cross-talk calibration scan sequentially reads out each pixel while charge is injected from the front end chip into the two adjacent pixels on both long sides. On scanning the applied bias voltage $V_{\text {bias }}$, a transition is observed from the low voltage, partially depleted region where there is a high ohmic short between pixels, to the high voltage, fully depleted region when the pixels are isolated from each other. The change in cross-talk is used to determine the depletion voltage. The injected charge is chosen such that cross-talk hits are seen only for $\mathrm{V}_{\text {bias }}<\mathrm{V}_{\text {pinch-off }} \sim \mathrm{V}_{\text {FullDepletion }}$.

The resulting depletion voltage curve averaged over all modules in the innermost Pixel Detector barrel layer is shown in Figure 7(a) for cross-talk scans taken with the parameters in Table 7(b). A significant decrease in the depletion voltage is observed between the scans on 11 May and 17 June, as expected before type-inversion due to the increase in integrated luminosity. The rise in the

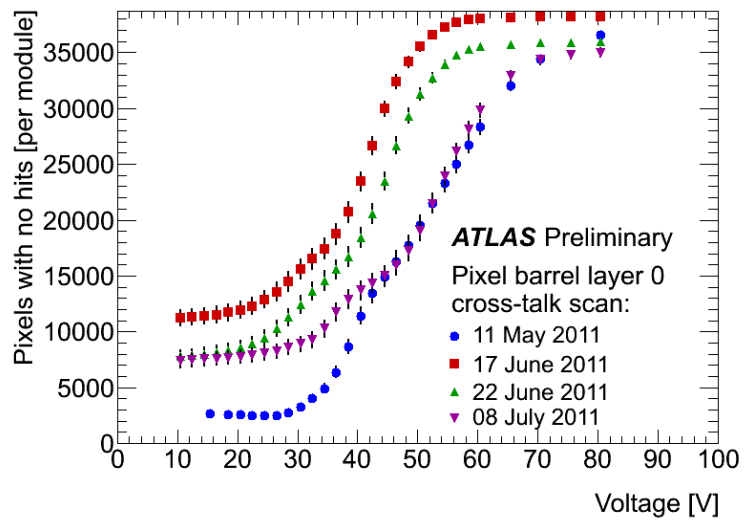

(a)

\begin{tabular}{|c|c|c|}
\hline $\begin{array}{c}\text { Cross-talk } \\
\text { scan date }\end{array}$ & $\begin{array}{c}\text { Integrated } \\
\text { luminosity }\end{array}$ & $\begin{array}{c}\text { Injected } \\
\text { charge (e) }\end{array}$ \\
\hline \hline 11 May 2011 & $267.5 \mathrm{pb}^{-1}$ & $2 \times 60000$ \\
17 June 2011 & $1056.5 \mathrm{pb}^{-1}$ & $2 \times 60000$ \\
22 June 2011 & $1107.4 \mathrm{pb}^{-1}$ & $2 \times 80000$ \\
\hline \multicolumn{3}{|c|}{ Detector cooling system stoppage } \\
\hline 8 July 2011 & $1286.5 \mathrm{pb}^{-1}$ & $2 \times 80000$ \\
\hline
\end{tabular}

(b)

Figure 7: Number of pixels missing hits vs applied bias voltage (a) for a series of cross-talk scans (b). 
number of pixels with no hits at low voltage between these first two scans suggests an increase in the resistance between adjacent pixels. The injected charge was increased in the scan on 22 June to reduce the number of pixels with no hits, which causes an artificial rise in the depletion voltage. The 8 July scan taken after a detector cooling system stoppage indicates beneficial annealing.

\subsection{Track based depletion depth studies}

A track based method has been developed to estimate the depth of the depleted zone in the sensor. The interpolated entry point of the reconstructed track and its incidence angle at the module surface are used to estimate the depth of the track segment below each pixel in a cluster. In an extension to earlier work [5] the method has been applied in the long pixel direction, to reduce Lorentz angle effects and to ensure a large range of incident angles from collision events.

The measured track depth distribution is shown for different cluster widths in Figure 8(a). Ideally, this would be limited by the depth of the depleted region; in practice the boundaries of the distribution are smeared by cross-talk and threshold effects, which depend on the incidence angle of the track. In order to correct for angle-dependent effects, the distribution is divided into slices

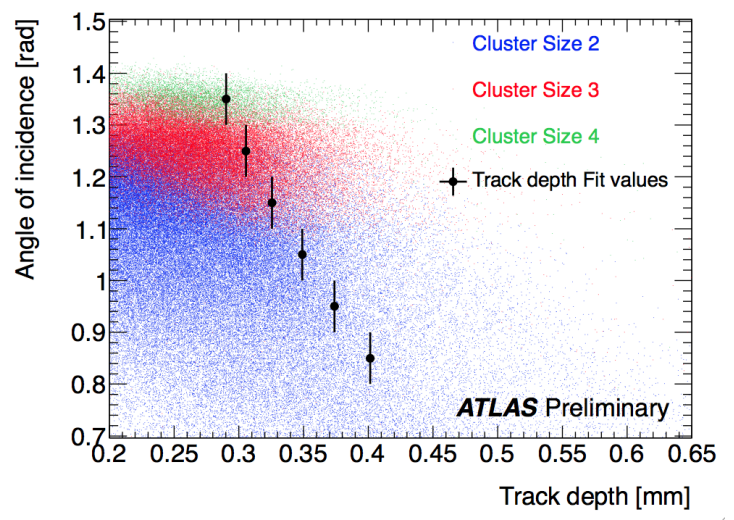

(a)

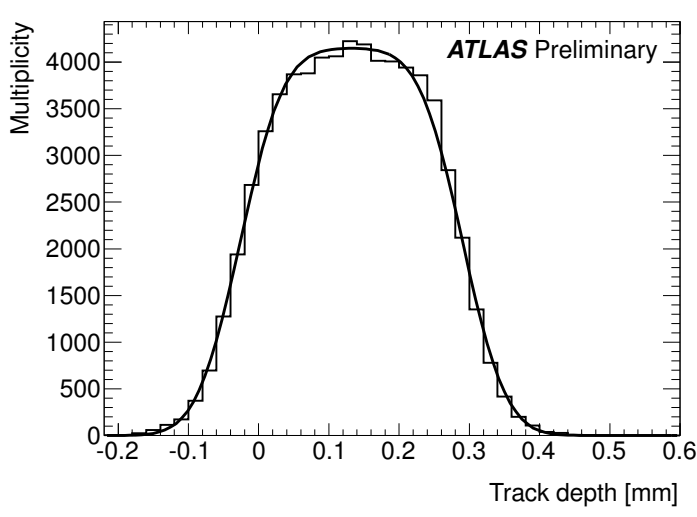

(b)

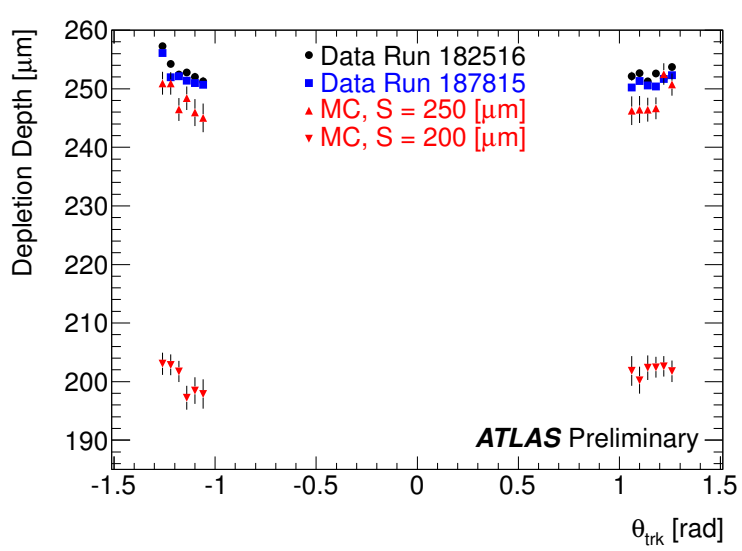

(c)

Figure 8: (a) Angle of incidence in the long pixel dimension vs track depth for different cluster widths, overlaid by the result of an error function fit in angular slices. (b) The track depth distribution for the slice $1.3 \leq \theta_{\text {trk }}<1.4$ [rad]. (c) The depletion depth for each angular slice after threshold correction, shown for 2011 ATLAS data and Monte Carlo simulations 
for various ranges of the angle of incidence. The distribution for one such angular slice is shown in Figure 8(b). The depletion depth is determined independently through a fit to each angular slice, corrected using the information from the left boundary of the distribution, corresponding to lower track depths.

In the calculation of the track depth distribution, the depletion depth is initially assumed to be equal to the nominal sensor thickness. This assumption is corrected by exploiting the charge deposited for different track entry points to locate the edge of the pixel. The required correction is then determined from the pixel edge position, the track incidence angle and the known pixel length of $400 \mu \mathrm{m}$, which provides the length scale for the measurement.

After all corrections, the depletion depth is measured by averaging the values for all angular slices, shown for data and simulation in Figure 8(c). The depletion depths are determined for two 2011 data sets taken at nominal $150 \mathrm{~V}$ bias voltage: $D_{182516}=253 \pm 3$ (stat.) \pm 10 (sys.) $\mu \mathrm{m}$ and $D_{187815}=252 \pm 2$ (stat.) \pm 10 (sys.) $\mu \mathrm{m}$. The measurements are in good agreement with the nominal $250 \mu \mathrm{m}$ thickness of the active sensor, indicating that the sensor is fully depleted. Checking the method using two Monte Carlo samples with input depletion depths of $250 \mu \mathrm{m}$ and $200 \mu \mathrm{m}$ shows good agreement: $D_{M C 250}=248 \pm 8$ (stat.) \pm 10 (sys.) $\mu \mathrm{m}$ and $D_{M C 200}=201 \pm 6$ (stat.) \pm 10 (sys.) $\mu \mathrm{m}$. The method is suitable after type-inversion for future monitoring of the depletion depth.

\section{Conclusion}

A careful monitoring program has observed the predicted, early effects of radiation damage in the ATLAS silicon tracker, including a clear increase in leakage current in the pixel and microstrip sensors in proportion to the fluence. First comparisons of data with simulation show good agreement at the level of $\sim 10 \%$ in the barrel regions. The latest bias scans reveal the depletion voltage is reduced in the pixel sensors before type-inversion and indicate that the cross-talk is reduced between under-depleted, adjacent pixels. A track-based method was presented to measure the depletion depth after type-inversion, enabling continuous monitoring of the sensor performance.

\section{Acknowledgments}

We congratulate the LHC accelerator team for a magnificent and timely increase in luminosity, which irradiated the ATLAS silicon tracker enough for initial damage effects to be observable.

\section{References}

[1] The ATLAS Collaboration, The ATLAS Experiment at the CERN Large Hadron Collider, JINST 3 (2008) S08003.

[2] I. Gorelov et al., ATLAS Pixel Radiation Monitoring with HVPP4 System, arXiv:0911.0128v1.

[3] A.Chilingarov, Generation current temperature scaling, RD50 Technical Note, RD50-2011-01.

[4] R.S. Harper et al., Evolution of silicon microstrip detector currents during proton irradiation at the CERN PS, Nucl. Instr. Meth. A 479 (2002) 548-554.

[5] I. Gorelov et al., A measurement of Lorentz angle and spatial resolution of radiation hard silicon pixel sensors, Nucl. Instr. and Meth. A, 481:204-221, 2002. 